\title{
Der Raumordnerische Vertrag als Instrument zur Absicherung von Kooperationen im zentralörtlichen System
}

\author{
The Spatial Planning Contract \\ as an Instrument to Secure Co-operations \\ in the Central Place System
}

\begin{abstract}
Kurzfassung
Der Beitrag widmet sich der Frage, inwieweit Kooperationen das zentralörtliche System als Konzept der Raumordnung stärken können, dessen Legitimation als Teil der klassischen hoheitlich agierenden Raumordnung in Zweifel gezogen wird. Das Hauptaugenmerk wird dabei auf die Anwendung des Funktionalprinzips statt des gängigen Territorialprinzips bei der Zuordnung zentralörtlicher Funktionen gelegt. Die Steuerungswirkung der diesbezüglich in der raumordnerischen Praxis erprobten Städteverbünde bzw. gemeinsamen zentralen Orte leidet unter der mangelnden verbindlichen Festlegung einer Funktionsteilung. Hier bringt der Verfasser den raumordnerischen Vertrag als Lösungsweg ins Spiel und diskutiert dies am Beispiel des Raumes Dessau.
\end{abstract}

\begin{abstract}
The article considers the question to what extent co-operations can strengthen the central place system as a concept of spatial planning. The legitimacy of the central place system as part of classical spatial planning that acts in a sovereign way is questioned. Here the focus is put on the application of the principle of functionality instead of the conventional principle of territoriality in the allocation of central place functions. The policy impacts of urban associations and shared central places that have been tested in spatial planning practice in this respect suffer from the lack of a binding agreement on the distribution of functions. In this context the author introduces the spatial planning contract as a possible solution and discusses it using the example of the Dessau area.
\end{abstract}

\section{Einfiihrung}

Das zentralörtliche System wird in seiner klassischen Ausprägung zunehmend in Frage gestellt. Dies bezieht sich sowohl auf reale Steuerungsdefizite als auch die grundsätzliche Abkehr von der klassischen, ordnungsrechtlich ausgerichteten Raumordnung hin zu diskursiven Formen planerischen Handelns. Vor diesem Hintergrund erscheint das System zunächst eher hinderlich, da es die eigene Handlungsflexibilität - etwa im Rahmen sogenannter „Städtenetze“ - einschränkt. Gleichzeitig erkennen aber auch seine Kritiker die Notwendigkeit einer Steuerung der Siedlungstätigkeit an, nunmehr vor dem Hintergrund der Leitvorstellung einer nachhaltigen Raumentwicklung.
Im vorliegenden Beitrag wird zunächst das zentralörtliche System als Konzept der Raumordnung erläutert, um dann auf seine in der Anwendung zu beobachtenden Defizite und sein Verhältnis zu diskursiven Formen planerischen Handelns einzugehen. Auf dieser Grundlage werden Überlegungen zur Instrumentalisierung des zentralörtlichen Systems für ein kooperatives Verwaltungshandeln unter Orientierung am Funktionalprinzip angestellt. Im Mittelpunkt steht dabei die Anwendung des sogenannten „Raumordnerischen Vertrages“ nach $\S 13$ Satz 5 ROG zur verbindlichen Absicherung einer zuvor zwischen Landesplanung und beteiligten Kommunen ausgehandelten Funktionsteilung eines Städteverbundes am Beispiel Dessau-Roßlau-Aken. 


\section{Das zentralörtliche System als Konzept der Raumordnung}

Das zentralörtliche System stellt zunächst eine siedlungsstrukturelle Normenvorgabe dar, die im Wesentlichen schon in den 1960er und 1970er Jahren in die Landesplanung implementiert und den 1990er Jahren in den neuen Bundesländern übernommen worden ist. Diese fußt zum einen auf deskriptiven Kriterien, und hier im Wesentlichen Verflechtungskriterien, und zum anderen auf normativen Kriterien, also dem erklärten Willen des Normgebers, eine bestimmte Siedlungsstruktur anzustreben bzw. im Umkehrschluss abweichende Entwicklungen zu verhindern. Dies kann auch bedeuten, dass Gebietskörperschaften Zentralitätsstufen zuerkannt werden, die sie im Hinblick auf die vom Gesetzgeber für maßgeblich erachteten deskriptiven Kriterien noch nicht oder nicht mehr erfüllen, deren Erfüllung aber aus raumordnungspolitischen Gründen für erforderlich erachtet wird. Daher ist mit dem System immer auch eine politische Willensbekundung - oder negativ ausgedrückt - „Wunschvorstellung" verbunden.

Insofern unterscheidet sich das zentralörtliche System als raumordnerisches Konzept sowohl von der regionalökonomischen Zentrale-Orte-Theorie, die auf Christaller zurückgeht, als auch von empirisch beschreibbaren zentralörtlichen Systemen in der Realität. ${ }^{1}$

Das zentralörtliche System wird als Teil der klassischen, regulierenden Raumórdnung begriffen. Es erlaubt auf der Grundlage „rationaler“ empirischer Untersuchungen eine politisch neutrale Einstufung der Städte und Gemeinden eines Landes in ein System zentraler Orte durch eine zentrale raumordnerische Instanz. ${ }^{2}$

Dennoch lassen sich unstrittig auch Wirkungen des Systems empirisch nachweisen. Insbesondere über den Vergleich mit Ländern, die dieses System zur Steuerung der Zentrenstruktur nicht verfolgt haben (z.B. Frankreich, Italien, Belgien), aber auch bei einem Blick auf die fatalen Entwicklungen der neuen Bundesländer, die in den ersten Jahren nach der Wiedervereinigung ohne raumordnerisches Steuerungsinstrumentarium gerade im Bereich der Siedlungsentwicklung immense Fehlentwicklungen zu verzeichnen hatten. Dies gilt insbesondere für Faktoren, die zum klassischen Steuerungsbereich des zentralörtlichen Systems gehören, nämlich den Einzelhandel sowie die Infrastruktur. $^{3}$

Ohne ein siedlungspolitisches Ordnungsmodell wie das zentralörtliche System würden zweifellos der Entscheidungsspielraum, und damit die Flexibilität auf aktuelle Entwicklungen angemessen reagieren zu können, im Einzelfall wachsen. Dem steht aber eine $\mathrm{Zu}$ nahme politisch motivierter Aushandlungsprozesse gegenüber, die sich an keinen Vorgaben mehr orientieren könnten, womit auch abstrakte raumordnungspolitische Zielvorstellungen wie die Leitvorstellung der nachhaltigen Entwicklung hinfällig wären. ${ }^{4}$ Besonders im Hinblick auf die Standortentwicklung des großflächigen Einzelhandels und der so genannten „Factory Outlet Center" und im Hinblick auf flächen- und verkehrssparende Siedlungsstrukturen, erscheint eine auch rechtlich verbindliche Operationalisierung der Leitvorstellung unverzichtbar.

In der planerischen Praxis haben sich aber immer deutlicher Defizite in der faktischen Steuerungsfähigkeit des starren Systems gezeigt, das Gegenstand langfristig angelegter Pläne ist, denen sich immer schneller verändernde sozioökonomische Rahmenbedingungen gegenüber stehen. Außerdem hat auch die mangelnde Umsetzungsorientierung der Raumordnung dazu geführt, dass zentralörtliche Funktionszuweisungen häufig reine Wunschvorstellungen geblieben sind, da sich die Akteure, die umsetzungsbefähigt waren, entweder nicht an die Zielfestlegungen der Landesplanung gebunden fühlten (z.B. andere Ressorts wie Finanz-, Verkehrs- oder Städtebauministerien) oder angesichts der schlichten Unkenntnis bzw. mangelnden Bindungswirkung gegenüber Privaten nicht in ihre Entscheidungen einbezogen haben (z.B. Standortsuche von Unternehmen). Teilweise wird auch der fehlende Entwicklungsbezug kritisiert und von einer „Konservierung“ bestehender Strukturen über die Funktionszuweisungen des zentralörtlichen Systems gesprochen. ${ }^{5}$ Zudem bestehe jedenfalls nicht in dem Ausmaß, wie es das System unterstellt, ein eindeutiger Konsumentenbezug zu bestimmten Zentralen Orten, die in räumlicher Nähe liegen. Vielmehr werden häufig Güter und Dienstleistungen an unterschiedlichen Orten nachgefragt, wobei als Entscheidungskriterium von einer Zeit-Kosten-Mühe-Relation auszugehen ist. ${ }^{6}$ Verstärkt wird diese Tendenz zur Mehrdimensionalität noch durch die teilweise „Enträumlichung“ der Angebote über Versandhandel und zunehmend das Internet.

Zudem verlieren angesichts der wachsenden Dynamik und Heterogenität unserer Gesellschaft hoheitlich normative Konzepte mit Langfristcharakter wie das zentralörtliche System tendenziell ihre Funktion zur Erreichung raumordnungspolitischer Zielvorstellungen zugunsten der Moderation von regionalen Konsensfindungsprozessen und dem Management konkreter umsetzungsfähiger Projekte. 


\section{Kooperative Elemente im zentralörtlichen System}

Im Folgenden gilt es zu prüfen, inwieweit sich dieses ordnungspolitische Erfordernis mit neueren, am Kooperationsgedanken ausgerichteten raumplanerischen wie verwaltungswissenschaftlichen Vorstellungen in Einklang bringen lässt. Damit sind die Zielvorstellungen einer einerseits an die Entwicklungsdynamik unserer Gesellschaft angepassten Flexibilisierung und andererseits in seiner faktischen Bindungswirkung durch die Beteiligung Privater gestärkten zentralörtlichen Systems verbunden.

Insbesondere in Verdichtungsräumen ist ein spezifisches Kooperationserfordernis im Hinblick auf das Angebot zentralörtlicher Versorgungsdienstleistungen als auch bei der Entwicklung zentralörtlicher Funktionen im Bereich Arbeiten, Wohnen, Einzelhandel, Freizeit und Verkehr gegeben. Dies begründet sich mit dem Umstand, dass die bisherigen Versuche, diese Probleme mittels klassischer zentralörtlicher Funktionszuweisungen an einzelne Standorte bzw. Städte zu lösen, wenig erfolgreich gewesen sind. Die wesentliche Funktion des zentralörtlichen Systems, nämlich die Sicherstellung einer wohnortnahen Versorgung mit Gütern und Dienstleistungen, tritt hier in den Hintergrund. Prägend sind Konkurrenzen in der Versorgungsfunktion zwischen Grund- und Mittelzentren einerseits und Kernstädten andererseits, wobei sich die Einzugsbereiche stark überschneiden. Von einem eigenständigen auszuweisenden Einzugsbereich kann daher nicht die Rede sein. So gesehen ist es wenig einsichtig, warum die Allokation von raumbedeutsamer Infrastruktur oder auch Einzelhandel an der Stadtgrenze eines zentralen Ortes Halt machen muss, obwohl Umlandgemeinden u.U. über geeignetere Standorte verfügen und eine Einigung mit dem zentralen Ort erzielbar ist.

Auf konzeptioneller Ebene wird insbesondere die Schaffung oberzentraler Funktionsräume („Funktionalprinzip") angeregt, die von gemeindespezifischen Aussagen ("Territorialprinzip“) Abstand nehmen. ${ }^{7}$ In eine ähnliche Richtung zielen Zentrenverbünde, wie sie auch in der Sächsischen Landesplanung bereits im Zusammenhang mit der Abkehr von auf Einzelstandorte bezogenen Versorgungskonzepten praktiziert werden. Ebenfalls am Funktionalprinzip orientieren sich Überlegungen von Winkel, der „kooperative Umlandverbünde" und die Ausweisung von „zentralörtlichen Funktionsräumen" anregt. ${ }^{8}$

Die mit derartigen Funktionsräumen verbundene $\mathrm{Zu}$ sammenarbeit der beteiligten Gebietskörperschaften soll auf kooperativer Basis erfolgen. Voraussetzung zur Bildung derartiger Kooperationen im Siedlungsgefüge sind regelmäßig Übereinstimmungen zwischen den Beteiligten hinsichtlich Problemsicht und Lösungswegen. Das Handeln ist auf Freiwilligkeit und Gleichberechtigung ausgerichtet und zumindest zunächst rein informell, obwohl später nach Erzielung eines stabilen Konsenses das Bedürfnis nach Absicherung der erzielten Übereinkunft wachsen kann. So gesehen sind formale Organisationen und Rechtsformen als Resultante, nicht jedoch als Voraussetzung abgestimmten Handelns anzusehen.

Das zentralörtliche System als raumordnerisches Konzept ist dabei in der Lage, bei der Konsensfindung über Problemwahrnehmung und Problemdefinition in diskursiven Prozessen eine maßgebliche Rolle zu spielen. Es ist unmittelbar plausibel und in der politisch-planerischen Kommunikation etabliert als Werkzeug zur Beschreibung bestehender und Darstellung politisch gewollter Siedlungsstrukturen, das sich auch graphisch verständlich visualisieren lässt. ${ }^{9}$

Gleichwohl muss nicht automatisch die Zielrichtung der kooperierenden Städten im Hinblick auf die räumliche Struktur mit der aus einer regionalen Perspektive übereinstimmen. So werden auch Konfliktbereiche zwischen zentralörtlichem System und dem Konzept der Städtenetze gesehen, insbesondere im Hinblick auf eine Aufteilung von Versorgungsfunktionen und damit einhergehender ökologisch kontraproduktiver Raumentwicklungen (größeres Verkehrsaufkommen) ${ }^{10}$ bzw. mögliche Nachteile für ohnehin raumstrukturell benachteiligte Städte, die aus den Kooperationen ausge klammert bleiben könnten, angeführt."

Im Übrigen eignen sich freiwillige Kooperationen vor allem zur Abstimmung räumlichen Handelns, wenn eine Win-Win-Situation vorliegt. Konkurrenzen um Entwicklungsperspektiven, um die Verteilung knapper Ressourcen zwischen den beteiligten Städden und Gemeinden bleiben ungelöst, wie auch die Autoren der ARL-Schrift einräumen, wenn sie ausführen, dass eine enge interkommunale Abstimmung über die räumlicher Verteilung zentralörtlicher Funktionen erforderlich sei. ${ }^{12}$

Kooperationen im Zusammenhang mit dem zentralörtlichen System sind also nichts Neues oder Ungewöhnliches. Die Frage ist jedoch, ob diese auch Eingang in das raumordnerische Zentrale-Orte-Konzept eines Landes finden und so zu verbindlichen Handlungsnormen werden - also ein horizontaler Konsens zwischen den Gebietskörperschaften in einer Region durch einen vertikalen zwischen Kommunen und Landes- bzw. Regionalplanungsträger ergänzt werden kann. Diese verbindliche Funktionsteilung hat in der Vergangenheit bei der Festlegung gemeinsamer zen- 
traler Orte, Städteverbünde oder anderer Formen interkommunaler Zusammenarbeit gefehlt. Damit wurde das eigentliche Ziel, nämlich eine Kooperation statt interkommunaler Konkurrenz, verfehlt, weil jede beteiligte Gebietskörperschaft weiterhin mit Recht jedwede zentralörtliche Funktion für sich einfordern konnte. Umgekehrt sind bisher rein informelle $\mathrm{Ab}$ sprachen zwischen Gebietskörperschaften über eine Zusammenarbeit bei der Vorhaltung zentralörtlich bedeutsamer Infrastruktur oder in Fragen der Siedlungsentwicklung nicht in das Zentrale-Orte-Konzept eingeflossen.

In beiden Fällen kommt nach Auffassung des Verfassers der raumordnerische Vertrag als ideales Bindeglied zwischen hoheitlichem und rein freiwilligem und informellem Handeln ins Spiel, auf den nachfolgend näher eingegangen werden soll.

\section{Raumordnerische Verträge als Möglichkeit für kooperatives Verwaltungshandeln}

Im Folgenden wird der Raumordnerische Vertrag als Instrument und hinsichtlich seiner rechtlichen Implikationen zunächst näher erläutert, bevor mit Hilfe eines Anwendungsbeispiels die Praktikabilität des Ansatzes aufgezeigt wird.

\section{Der Raumordnerische Vertrag}

$\S 13$ Satz 5 ROG sieht vor, dass vertragliche Regelungen zur Vorbereitung und Verwirklichung von Raumordnungsplänen getroffen werden können.

Damit geht ihr mögliches Einsatzfeld zwar weit über den hier relevanten Bereich der Lösung von StadtUmland-Problemen hinaus, doch vielfach ist ein interkommunaler Interessenausgleich Voraussetzung dafür, dass die Landesplanung ihre Ziele umsetzen kann. Im Gegensatz zu städtebaulichen Verträgen gemäß $\$ 11$ BauGB ist hier auch das Land Vertragspartner, womit nicht nur eine horizontale Abstimmung zwischen Kommunen, sondern auch eine vertikale zwischen Land und Kommunen ermöglicht wird. Denkbar sind nach Aussage von Runkel (BMVBW): „Vereinbarungen im Zusammenhang mit der Festsetzung bzw. Erreichung von Zentralitätsstufen." Spannowsky führt aus, dass „Kooperationsverträge zur Bewältigung von Funktionskonflikten im zentralörtlichen Gliederungssystem und zur Fortschreibung der zentralörtlichen Gliederung" und „Vereinbarungen zwischen dem Träger der Raumordnung und Gemeinden $[\ldots]$ zur Lösung funktionsräumlicher Stadt-Umland-Probleme $[\ldots]$ " denkbare Einsatzfelder für raumordnerische Verträge darstellen können. ${ }^{13}$
Der raumordnerische Vertrag bietet aufgrund seiner großen Flexibilität die Möglichkeit, eine maßgeschneiderte Lösung für ein Stadt-Umland Problem in relativ kurzer Zeit freiwillig und kooperativ fixieren zu können. In der Regel wird dem eine Phase informeller Absprachen vorangehen, in der eine grundsätzliche Úbereinstimmung über bestimmte Zielvorstellungen herbeigeführt wird. An deren Ende kann der Wunsch nach Formalisierung des erzielten Konsenses stehen. Hier in Form eines freiwilligen, zwei- oder mehrseitigen Rechtsgeschäfts (dem Vertrag), der gleichwohl nach Unterzeichnung eine starke Bindungswirkung gegenüber den Vertragspartnern begründet.

Informelle $A b s p r a c h e n$ reichen für eine verlässliche Kooperation nicht aus, da sie häufig personenabhängig sind und beim Wechsel dieser Personen, sei es durch eine Änderung politischer Mehrheiten oder schlicht infolge des Erreichens der Altersgrenze, häufig nicht mehr gelten.

Wenn die Gemeinden allein im Rahmen eines interkommunalen Interessenausgleichs in der Lage wären, $z u$ einer Übereinkunft zu kommen, bedürfte es keines Raumordnerischen Vertrags. Dies ist jedoch bei Fragen bezüglich des zentralörtlichen Systems nicht der Fall, da eine Abstimmung mit der Landes- und Regionalplanung erforderlich ist. Andere institutionalisierte Formen der Zusammenarbeit wie etwa Zweckverbände, an dem sich auch andere Planungsträger und damit auch die Landesplanung beteiligen können, sind wiederum wesentlich aufwändiger als bloße Vertragswerke. Es entstünde eine eigene Körperschaft des öffentlichen Rechts mit Vorstand, Verbandsversammlung und in der Regel eigenem Personal, während im Rahmen der Regelungen, die Gegenstand eines Raumordnerischen Vertrages sind, allein auf die bestehenden Kapazitäten der Beteiligten zurückgegriffen wird.

\section{Rechtliche Implikationen des Raumordnerischen Vertrags}

Da bei Raumordnerischen Verträgen in der Regel Vereinbarungen über raumplanerische Sachverhalte beschlossen werden, handelt es sich um öffentlichrechtliche Verträge. Sie dienen dem Vollzug einer gesetzlichen Regelung, die dem öffentlichen Recht angehört. Raumordnerische Verträge können sowohl koordinationsrechtliche als auch subordinationsrechtliche Verträge sein. Dies hängt von den Vertragsparteien, ihrem rechtlichen Verhältnis zueinander und den Regelungsinhalten im Einzelfall ab.

Beim Raumordnerischen Vertrag sind die üblichen rechtlichen Grenzen bei öffentlich-rechtlichen Verträgen auf der Grundlage der $\$ \S 54$ ff. VwVfG zu beach- 
ten. Der Vertrag als solcher gilt nur inter partes. Zulässig sind auch Vertragsinhalte, die in einem formellen Plan nicht festsetzungsfähig wären. Dies gilt insbesondere für die Konkretisierung bzw. den Vollzug von Planungsinhalten. Dabei bezieht sich die Bindungswirkung des Vertrages nicht auf $\$ 4$ ROG, sondern auf die vertraglichen Regelungen, wie etwa Handlungs- und Erfüllungspflichten der Beteiligten, an deren Nichteinhaltung bestimmte Sanktionen geknüpft werden können. ${ }^{14}$

Im Bereich der Umsetzung bereits fixierter raumordnerischer Ziele können Raumordnerische Verträge für eine vorhabenbezogene Konkretisierung dieser Zielvorstellungen sorgen und damit die Landes- bzw. Regionalplanung in den Umsetzungsprozess abstrakter raumordnerischer Zielvorstellungen einbeziehen. Bei zielkonkretisierenden Vereinbarungen liegt ein koordinationsrechtlicher Vertrag vor, da das Druckmittel einer alternativen einseitigen Regelung fehlt. ${ }^{15}$

Die Verbindlichkeit der Verträge kann erhöht werden, indem die Landes- bzw. Regionalplanung die vertraglich vereinbarten Ziele als Ziele der Landesplanung in Raumordnungspläne aufnimmt, womit zusätzliche Bindungswirkungen entstehen, weil alle öffentlichen Planungsträger strikt daran gebunden sind ( $\$ 4$ Abs. 1 ROG). Ein planersetzender Vertrag, das heißt Vertragsinhalte mit der Bindungswirkung von Zielen der Raumordnung zu versehen, ist nicht denkbar, da materielle Rechtsnormen nicht durch Verträge ersetzt werden dürfen. ${ }^{16}$

\section{Anwendungsbeispiel gemeinsames Oberzentrum Dessau-Roßlau-Aken}

Das folgende Beispiel soll zeigen, dass sich der vorgestellte Ansatz eines Raumordnerischen Vertrages im Zusammenhang mit der Bewältigung von Funktionskonflikten im zentralörtlichen Gliederungssystem in der Praxis grundsätzlich realisieren lässt.

Der Vorschlag ist im Zusammenhang mit einem Gutachten zur kommunalen Gebietsreform in SachsenAnhalt entstanden, dessen Mitverfasser der Autor des vorliegenden Beitrags war. ${ }^{17}$ Ziel war es, mittels der Schaffung eines gemeinsamen Oberzentrums und der Vereinbarung einer Funktionsteilung im Rahmen eines Raumordnerischen Vertrages die Eingemeindung der Umlandgemeinden in das Oberzentrum zu vermeiden. ${ }^{18}$ Diese Konstruktion stimmt in weiten Teilen dem Modell der „Kooperativen Umlandverbünde“, auch wenn hier vordergründig drei Städte unterschiedlicher Zentralitätsstufe kooperieren und nicht auch Umlandgemeinden ohne Zentralitätseinstufung. Dies relativiert sich, wenn man berücksichtigt, dass im Fall Roß- lau mehrere kleinere Umlandgemeinden in die Stadt eingemeindet werden sollten und in einem Fall (Rodleben) auch zentralörtliche Funktionen (Güterverkehrszentrum) übernehmen. ${ }^{19}$

Zum Hintergrund des Vorschlags gilt es Folgendes anzumerken: Erklärtes Ziel der damaligen Landesregierung war die Stärkung der oberzentralen Funktion der Stadt Dessau, die mit nur noch gut 80000 Einwohnern drohte, ihre Kreisfreiheit und ihren zentralörtlichen Status zu verlieren. Eine Besonderheit der auf Seite 376 in Abbildung 1 dargestellten Region besteht darin, dass bestimmte oberzentrale Teilfunktionen im Bereich Verkehr (Hafen, Güterverkehrszentrum) von benachbarten Städten (Roßlau, 14000 EW, Grundzentrum mit Teilfunktion Mittelzentrum, industrieller Schwerpunkt; Aken, $10000 \mathrm{EW}$, Grundzentrum) wahrgenommen werden.

Um das „schärfste Schwert“ des Kommunalrechts, die Eingemeindung zu vermeiden, wurde nun die Bildung eines gemeinsamen Oberzentrums angeregt. So sollte das im Landesraumordnungsplan festgelegte Oberzentrum Dessau, das auf Grund einer zu geringen Einwohnerzahl in Frage gestellt wurde, durch eine gemeinsame oberzentrale Aufgabenwahrnehmung mit den Städten Roßlau und Aken wesentlich gestärkt werden. Diese ergänzenden Teilfunktionen als industrieller Schwerpunkt (Roßlau) und Hafenstandort mit Containerterminal (Aken) soll insgesamt die oberzentrale Funktion Dessaus stärken. Demgegenüber sollte die Stadt Dessau natürlich die hauptsächlichen oberzentralen Funktionen weiter wahrnehmen.

Als Absicherung für die Kooperation wurde vom Gutachter ein raumordnerischer Vertrag im Sinne des $\$ 13$ Satz 5 ROG zwischen der Landesplanung, vertreten durch die oberste Landesplanungsbehörde, das Ministerium für Raumordnung und Umwelt sowie den Städten Dessau, Roßlau und Aken, vorgeschlagen. Im Rahmen des raumordnerischen Vertragswerkes sollte eine exakte Funktionsteilung zwischen den beteiligten $\mathrm{Ge}$ bietskörperschaften vereinbart werden, an der es in der Vergangenheit bei der Festlegung von zentralen Orten in Funktionsteilung gemangelt hat. Es konnte dabei nicht Ziel dieses Vertragswerkes sein, Roßlau und Aken aufgrund ihres Status als Mitglieder des gemeinsamen Oberzentrums eine zusätzliche Ausweisung von Wohnbauflächen oder Gewerbeflächen außerhalb ihrer Funktionswahrnehmung im Bereich Logistik zuzubilligen.

Ferner sollte zur Stabilisierung der Finanzkraft der Region, für die Dessau faktisch ähnliche Funktionen übernimmt wie Magdeburg und Halle für ihren oberzentralen Einzugsbereich, der gesamte Städteverbund 
Abbildung 1

Siedlungsstruktur im Raum Dessau

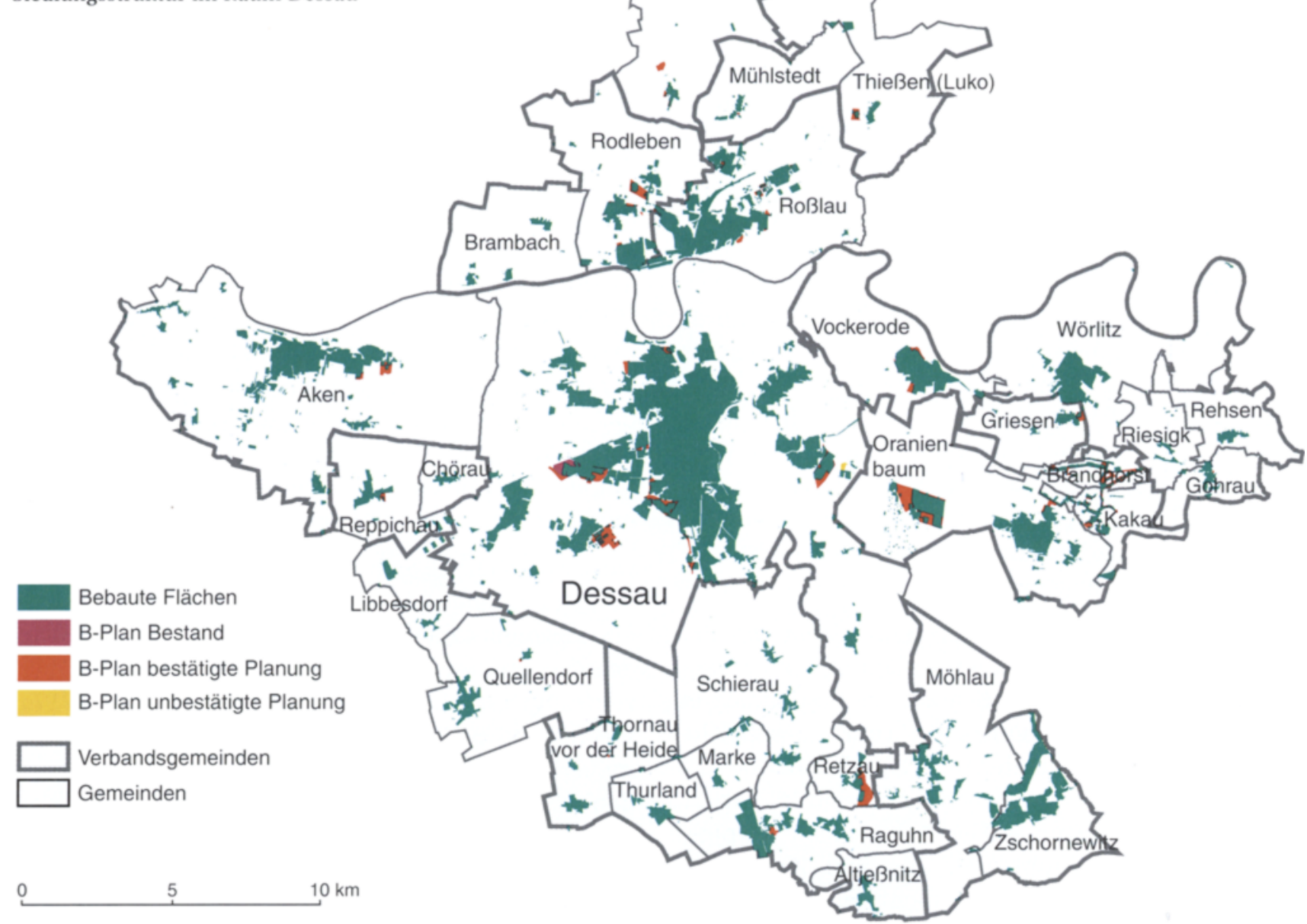

Quelle: Greiving; Turowski 2001, S. 144

im kommunalen Finanzausgleich als kreisfreie Stadt behandelt und so die Finanzausstattung der Region insgesamt verbessert werden. Damit würde der Städteverbund von der im Finanzausgleichsgesetz vorgesehenen überproportionalen Gewichtung ihrer Einwohner profitieren (27\% der allgemeinen Zuweisungen entfallen auf die kreisfreien Städte, obwohl diese nur $21,8 \%$ der Bevölkerung auf sich vereinen (Stichtag 30.6.1999). Dies sollte sowohl dazu beitragen, die finanziellen Probleme der Stadt Dessau als auch die relative Finanzschwäche der Städte Roßlau und Aken zu mindern.

Die exakte Verteilung der zusätzlichen Finanzmittel wäre Ergebnis eines politischen Aushandlungsprozesses gewesen, doch es gilt klarzustellen, dass Dessau aufgrund seiner überwiegenden Wahrnehmung überörtlicher Funktionen den Großteil der Gelder erhalten sollte, und zwar über seinen ungewichteten Bevölkerungsanteil am gemeinsamen Oberzentrum hinaus. Dabei ist zu berücksichtigen, dass insbesondere Roßlau ohnehin bereits von den Gewerbesteuereinnah- men, die sich aus ihrer Funktion als Schwerpunktstandort für Gewerbe ergeben, profitiert.

An dieser Stelle kann auch auf die von Hahne/von Rohr vorgeschlagenen Kooperationsfonds abgestellt werden, die aus Mitteln des kommunalen Finanzausgleichs gespeist werden sollen, die zuvor den Umlandzentren und der Kernstadt zur Wahrnehmung übergemeindlicher Aufgaben zugeflossen sind..$^{20} \mathrm{Im}$ Unterschied zu der negativen Beurteilung durch Kühl/ Busch, die die Kooperationsfonds für $\mathrm{zu}$ kompliziert einschätzen, um in die Verwaltungspraxis Eingang zu finden $^{21}$, wird hier eine andere Auffassung vertreten. Genau über diese Verteilung der Gelder hätte nämlich eine Einigung im Rahmen von $\$ 2$ des Raumordnerischen Vertrages getroffen werden sollen. Diese Überlegungen stehen im Einklang mit ökonomischen Überlegungen, die das Anreizproblem um so eher für lösbar erachten, je mehr man im kommunalen Finanzausgleich weg von pauschalierten ungebunden Finanzmitteln hin zu einem durch bestimmte zentralörtliche Leistungen verursachten Lastenausgleich kommt. ${ }^{22}$ 
Voraussetzung dafür wäre allerdings eine entsprechende Regelung im Gemeindefinanzierungsgesetz des jeweiligen Landes.

Weitere Vertragsbestandteile sollten im Übrigen die Beteiligung an einer gemeinsamen Teilraumentwicklungsplanung $(\S 1)$, eine gemeinsame Wirtschafts- und Kulturförderung ( $\$ 3$ ) und der koordinierte Ausbau der Verkehrsinfrastruktur $(\$ 4)$ sein.

Im Zuge der politischen Diskussion zur Umsetzung der Vorschläge zur Organisation der Stadt-Umland-Räume wurde der Vorschlag von den beteiligten Städten aufgriffen. Ein Vertragsentwurf mit Stand 20.2.2002 lag bereits vor (siehe Abb. 2), kam jedoch nicht mehr zur Unterzeichnung, weil im Ergebnis der Landtagswahl vom April 2002 die neue CDU/FDP Regierung auf die Umsetzung der von der Vorgängerregierung initiierten Gebietsreform verzichtet hat und damit die Hauptmotivation der beteiligten Städte, zu kooperieren, gegenstandlos wurde war. ${ }^{23}$

\section{Fazit}

Auch wenn das vorgestellte Beispiel nicht über das Stadium einer grundsätzlichen Übereinkunft der Beteiligten hinaus gelangt ist und erst aufgrund äußeren Drucks (drohende Eingemeindungen) Kooperationsbereitschaft erzeugt worden ist, so steht doch fest, dass das Instrumentarium eines Städteverbundes und die Absicherung mittels eines raumordnerischen Vertrages ein machbarer Weg zu einer interkommunal abgestimmten Entwicklung im Rahmen eines weiterentwickelten zentralörtlichen Systems darstellt. Es wird sicherlich zukünftig andere Fälle geben, in denen aufgrund bestehender Stadt-Umland-Probleme ein Konsens der Beteiligten über Problemsicht und Lösungsweg herstellbar ist und wo der hier vorgestellte Vorschlag hoffentlich eine konstruktive Anregung darstellen mag.

Insgesamt konnte gezeigt werden, dass das zentralörtliche System eine Zukunft als raumordnerisches Konzept besitzt, wenn kooperative Elemente aufgenommen werden und sich die Zuweisung von Zentralitätsstufen von Einzelstandorten löst. Dies darf freilich nicht dazu führen, dass jede Steuerungswirkung verloren geht, sondern muss stets von verbindlichen, ver- traglichen Vereinbarungen begleitet werden, die eine auch raumordnerisch sinnvolle und mit der Leitvorstellung einer nachhaltigen Raumentwicklung konforme Aufgabenverteilung absichert.

Ein gangbarer Weg wäre dabei, dass die Landesplanung ober- und ggf. auch mittelzentrale Funktionsräume festlegt und die Festlegung der Funktionswahrnehmung innerhalb dieses Raumes zum Gegenstand von Verhandlungen der beteiligten Gebietskörperschaften macht. Die Einigung darüber würde in einem raumordnerischen Vertrag verbindlich fixiert, der hinreichend flexibel ist, um bei geänderten Rahmenbedingungen eine Anpassung der Vertragsinhalte zuzulassen. 


\section{Anmerkungen}

(1) Blotevogel: Zum Verhältnis der regionalökonomischen Zentrale-Orte-Theorie zum Zentrale-Orte-Konzept der Raumordnung. In: Blotevogel (Hrsg.): Fortentwicklung des Zentrale-Orte-Konzepts. ARL. - Hannover 2002, S. 10

(2)

Danielzyk: Veränderter raumordnungspolitischer Kontext. In: Blotevogel (Hrsg.): Fortentwicklung des Zentrale-Orte-Konzepts. ARL. - Hannover 2002, S. 1 (6)

(3)

Blotevogel, a.a.O. (Fn. 1), S. 19

(4)

So auch Stiens/Pick: Die Zentrale-Orte-Systeme der Bundesländer. In: Raumforschung und Raumordnung 1998, S. 421 (432)

(5)

Winkel: Kooperative Umlandverbünde: Konzept der Zentralen Orte fortentwickeln. In: Der Landkreis 2000, S. 800

(6)

Deiters: Die Zentrale-Orte-Konzeption auf dem Prüfstand. In: Informationen zur Raumentwicklung 1996, S. 634

(7)

Blotevogel et. al.: Zentrale Orte in der künftigen Raumordnungspolitik. In: Blotevogel (Hrsg.): Fortentwicklung des ZentraleOrte-Konzepts. ARL.- Hannover 2002, S. 229

(8)

Winkel, a. a. O. (Fn. 3), S. 801, 803.

(9)

Blotevogel et. al., a. a. O (Fn. 7), S. 225

(10)

Stiens/Pick, a. a. O. (Fn. 6), S. 432

(11)

Priebs: Städtenetze als raumordnungspolitischer Handlungsansatz - Gefährdung oder Stütze des Zentrale-Orte-Systems? In: Erdkunde 1996, S. 35 (44)

(12)

Blotevogel et. al., a. a. O. (Fn. 7), S. 233

(13)

Spannowsky: Verwirklichung von Raumordnungsplänen durch vertragliche Vereinbarungen. BBR. - Bonn 1999; ders.: Raumordnerische Verträge als Instrument des kommunalen Interessenausgleichs. In: Gemeinsame Landesplanung Berlin/Brandenburg (Hrsg.): Kommunale und regionale Zusammenarbeit im Spree-Havel-Raum. - Berlin 2000. S. 38 (41)
(14)

Spannowsky, a. a. O. (Fn. 14), S. 10

(15)

Grotefels/Lorenz: Der landesplanerische Vertrag nach $\S 13 \mathrm{~S} .5$ ROG, UPR 2001, S. 328 (329)

(16)

BVerwG, Urteil vom 1.2.1980 - 4 C 40/77, NJW 1980, S. 2538f., Stüer. In: Per Vertrag zur fairen Landesplanung? Städte- und Gemeinderat 2000, S. 21 (22)

(17)

Greiving/Turowski: Untersuchung der Verflechtungsbeziehungen zwischen den kreisfreien Städten Magdeburg, Halle und Dessau und deren Umlandgemeinden. - Dortmund, Dezember 2001

(18)

Auch Winkel (a. a. O., Fn. 3, S. 804), präferiert zur Lösung von Suburbanisationsproblemen statt Eingemeindungen gemeindeübergreifende Kooperationen.

(19)

Dies steht nicht im Widerspruch zu der Motivation, Eingemeindungen zu vermeiden, da es sich um eigenständig nicht leistungsfähige Kleinstgemeinden ohne eigene Verwaltung handelte, die zudem baulich z. T. zusammen gewachsen sind.

(20)

Hahne/v. Rohr: Ansatzpunkte zur Weiterentwicklung des Zentrale-Orte-Systems Schleswig-Holsteins. In: Raumforschung und Raumordnung 1999, S. 143 (153)

(21)

Kühl/Busch: Gutachten zur Stadt-Umland-Problematik im Land Schleswig-Holstein. - Kiel 2001, S. 10

(22)

Postlep: Das Anreiz- und Ausgleichsproblem bei zentralörtlichen Leistungen und Möglichkeiten seiner Lösung. In: Blotevogel (Hrsg.): Fortentwicklung des Zentrale-Orte-Konzepts. ARL. - Hannover 2002, S. 37 (41)

(23)

Vereinbarung über die Zusammenarbeit zwischen dem CDU Landesverband Sachsen-Anhalt und dem FDP Landesverband Sachsen-Anhalt über die Bildung einer Regierungskoalition für die 4. Legislaturperiode des Landtags von Sachsen-Anhalt. Magdeburg 2002, S. 46, Regierungserklärung von Prof. Dr. Wolfgang Böhmer vom 20. Juni 2002. „Sachsen-Anhalt im Aufbruch - ein traditionsreiches Land mit Zukunft, S. 11

Dr.-Ing. habil Stefan Greiving

Universität Dortmund

Lehrstuhl Rechtsgrundlagen der Raumordnung

August-Schmidt-Straße 10

44227 Dortmund

E-Mail:stefan.greiving@uni-dortmund.de 